\title{
EFFECT OF SLIP ON POROUS-WALLED SQUEEZE FILMS IN THE PRESENCE OF A TRANSVERSE MAGNETIC FIELD
}

\author{
B. C. CHANDRASEKHARA, RAJANI KANTHA
} and N. RUDRAIAH

Dept. of Math., Central College

Bangalore Univ., Bangalore 560001 , INDIA

\begin{abstract}
The present investigation is devoted to study the effect of viscous resistance, arising due to sparse distribution of particles in porous media, on the load capacity and thickness time response of porous-walled squeeze films in the presence of a uniform magnetic field. The results of the analysis obtained by using Beavers and Joseph [1] slip-boundary condition show that the viscous resistance increases the load capacity and thickness time response of squeeze films when compared with the results of Chandrasekhara [2] obtained in the absence of viscous resistance. Hence, for efficient performance of a porous walled squeeze film a suitable porous media in which the material is loosely packed may be used.
\end{abstract}

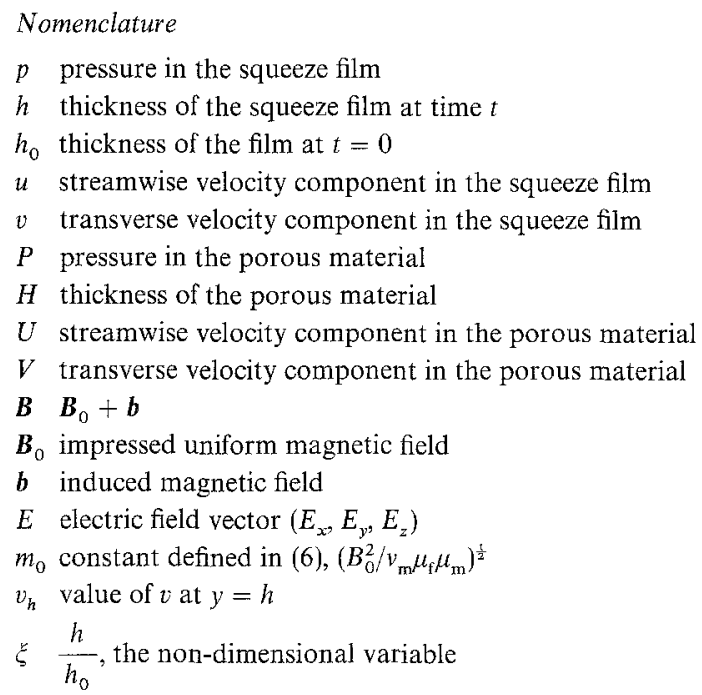


$\lambda_{n}$ eigen values

$\mu_{\mathrm{f}}$ viscosity

$\mu_{\mathrm{tm}}$ magnetic permeability

$\rho$ density

$v_{\mathrm{m}}$ magnetic diffusivity, $1 / \mu_{\mathrm{m}} \sigma_{\mathrm{e}}$

$\sigma$ dimensionless parameter, $\frac{h}{\sqrt{K}}$

$\sigma_{\mathrm{e}}$ electrical conductivity

$q$ velocity vector $(u, v)$

$L$ load capacity

$I_{n} \quad$ integral defined in (37)

$M$ Hartmann number defined in (7), $\left(m_{0}^{2} h^{2}\right)^{\frac{1}{2}}$

$l$ length of the strips in $x$-direction

$K$ permeability of the porous material

$J$ current density vector $\left(J_{x}, J_{y}, J_{z}\right)$

$t$ time

$G_{n}$ series coefficient appearing in equation (27)

\section{§ 1. Introduction}

In recent years there has been considerable interest in the study of the action of squeeze films between plane and cylindrical surfaces with and without magnetic field ([3]-[6]), with the object of designing more efficient bearings. These investigations are concerned with the film bounded by impermeable surfaces. However, if one of the walls is replaced by a permeable bed, Sparrow et al [7] have shown, using the Beavers and Joseph [1] slip boundary condition, that the load capacity of the film decreases and the thickness time response increases. Chandrasekhara [2] has extended this problem to a conducting flow and has shown that the presence of a uniform magnetic field increases the load capacity and response times of squeeze films.

The work of Sparrow et al [7] and Chandrasekhara [2] is related to the porous bed which is densely packed where the usual potential nature of Darcy law is valid. But to use the Beavers and Joseph [1] boundary condition, we note that at the nominal surface one has to use the boundary layer type of equation postulated by Brinkman [8] rather than the usual potential nature of the Darcy law. Recently Rudraiah [9] and Rudraiah and Nagaraj [10] have shown that in this case the boundary layer thickness is of order $K^{\frac{1}{2}}$. Therefore to study the squeeze film bounded by porous bed we have to use boundary layer type of equation where the solid particles are sparsely distributed rather than densely packed (see Taylor [11]). This is considered in this paper. Expressions for load capacity and 
thickness time relationship for the squeeze film are obtained. These expressions are numerically evaluated and the results are presented in graphs. Comparing the results of the present analysis with those of Chandrasekhara [2], we find that the squeeze film bearing made of a very porous material are more efficient than the squeeze film bearings made of less porous material.

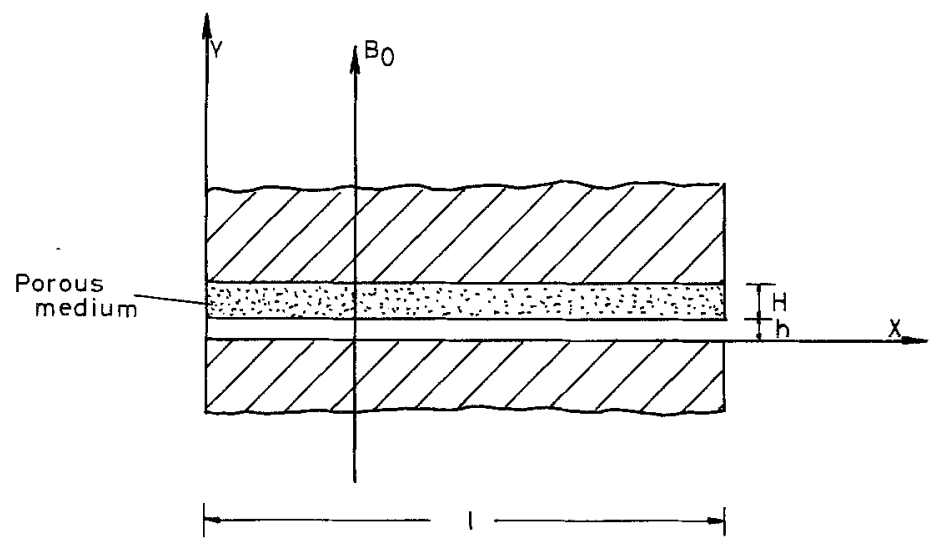

Fig. 1. Physical model.

\section{§2. Formulation of the problem}

The physical model (fig. 1) consists of two flat plates of the same dimensions separted by a gap, which is filled with a conducting, incompressible fluid of constant properties and of instantaneous height $h$. The fluid in the gap comprises the squeeze film. The lower plate is impermeable while the upper plate consists in part a porous material of constant thickness $H$. A uniform magnetic field $\boldsymbol{B}_{0}$ is applied transverse to the plates. Since the analysis is directed to study the combined effects of slip velocity and porous media on the load capacity of a squeeze film in the presence of a uniform magnetic field it is necessary to study the fluid motion both in the film and in the porous medium. For this we study the fluid flow in the film and in the porous media separately and the solutions are matched at the nominal surface.

\section{§3. Flow in the Squeeze Film}

To study the effect of magnetic field on the performance of squeeze films we consider a uniform magnetic field in the transverse direction. This assum- 
ption of uniform transverse magnetic field is valid because the thickness of the squeeze film that is encountered in practice is usually small. Therefore, the equations of motion which describe the fluid motion in the squeeze film under the assumption of laminar incompressible steady flow in the presence of a constant magnetic field $\boldsymbol{B}_{0}$ are:

$$
\begin{gathered}
\rho(\boldsymbol{q} \cdot \nabla) \boldsymbol{q}=-\boldsymbol{\nabla} p+\mu_{\mathrm{f}} \nabla^{2} \boldsymbol{q}+\boldsymbol{J} \times \boldsymbol{B} \\
\boldsymbol{\nabla} \cdot \boldsymbol{q}=0 \\
\boldsymbol{J}=\sigma_{\mathrm{e}}(\boldsymbol{E}+\boldsymbol{q} \times \boldsymbol{B}) \\
\boldsymbol{\nabla} \cdot \boldsymbol{B}=0 \\
\boldsymbol{\nabla} \times \boldsymbol{E}=0
\end{gathered}
$$

In accordance with the lubrication theory, the flow in the squeeze film is assumed to be dominated by viscous, lorentz and pressure forces, with inertia playing a secondary role. For many fluids used in the laboratory, the conductivity is usually small and hence the magnetic Reynolds number is very small, (ie. $R_{\mathrm{m}} \ll 1$ ). Therefore, we assume that the induced magnetic field is negligible compared to the applied magnetic field $\boldsymbol{B}_{0}$. Under the above assumptions, the equations of motion take the form (Chandrasekhara [2]):

$$
\frac{\mathrm{d}^{2} u}{\mathrm{~d} y^{2}}-m_{0}^{2} u=\frac{1}{\mu_{\mathrm{f}}} \frac{\partial p}{\partial x}+\frac{E_{z} \sigma_{\mathrm{e}} B_{0}}{\mu_{\mathrm{f}}}
$$

Equation (6) can be written in the form

$$
\frac{\mathrm{d}^{2} u}{\mathrm{~d} y^{2}}-\frac{M^{2}}{h^{2}} u=a_{0}
$$

where

$$
M^{2}=\frac{\sigma_{\mathrm{e}} B_{0}^{2} h^{2}}{\mu_{\mathrm{f}}}
$$

$u$ is the streamwise velocity component in the squeeze film, and

$$
a_{0}=\frac{1}{\mu_{\mathrm{f}}}\left(\frac{\partial p}{\partial x}+E_{z} \sigma_{\mathrm{e}} B_{0}\right)
$$


Equation (7) is solved using the boundary conditions:

$$
\begin{gathered}
u=0 \text { at } y=0 \\
-\frac{\mathrm{d} u}{\mathrm{~d} y}=-\frac{\alpha}{\sqrt{K}} u \text { at } y=h
\end{gathered}
$$

The condition (11) is the Beavers and Joseph [1] slip condition, $\alpha$ is the slip parameter and $K$ is the permeability of the porous medium expressed in units of length squared.

The solution of (7) satisfying (10) and (11) is

$$
\begin{aligned}
u=a_{0} h^{2} & {\left[\frac{\cosh (M y / h)-1}{M^{2}}-\right.} \\
& \left.-\left\{\frac{M \sinh M+\alpha \sigma(\cosh M-1)}{M^{2}(M \cosh M+\alpha \sigma \sinh M)}\right\} \sinh \left(\frac{M y}{h}\right)\right]
\end{aligned}
$$

where $\sigma=h / \sqrt{K}$, is a dimensionless number. We note that in the limit $M$ $\rightarrow 0$, equation (12) using (9) with $\boldsymbol{B}_{0}=0$, reduces to the Hydrodynamic case, namely

$$
u=\frac{h^{2}}{2 \mu_{\mathrm{f}}}\left[\left(-\frac{\partial p}{\partial x}\right)\left\{\left(\frac{y}{h}-\frac{y^{2}}{h^{2}}\right)+\frac{y / h}{1+\alpha \sigma}\right\}\right]
$$

The transverse velocity $v$ in the squeeze film at $y=h$, using the equations (12) and (2) is:

$$
v_{h}=-\frac{h^{3}}{\mu_{\mathrm{f}}} S \frac{\partial^{2} p}{\partial x^{2}}
$$

where

$$
S=\frac{M \sinh M(1-\alpha \sigma)-M^{2} \cosh M+2 \alpha \sigma(\cosh M-1)}{M^{3}(M \cosh M+\alpha \sigma \sinh M)}
$$

The pressure $p=P(x)$ appearing in the above equation is still an unknown. To determine this, it is necessary to have the knowledge of the flow conditions in the porous medium and is considered below. 


\section{§4. Flow in the porous medium}

The Beavers and Joseph [1] slip boundary condition is based on the existence of a boundary layer just below the nominal surface. This means that the void fraction near the nominal surface is somewhat larger compared to that away from the nominal surface. This means that the porosity near the nominal surface is large compared to that away from the nominal surface so that we have to take into account of the skin friction at the nominal surface. Therefore, following Rudraiah et al ([12], [13]), we have to use the boundary layer type of equation, namely

$$
O=-\nabla P+\mu_{\mathrm{f}} \nabla^{2} \boldsymbol{q}+\boldsymbol{J} \times \boldsymbol{B}-\frac{\mu_{\mathrm{f}}}{K} \boldsymbol{q} .
$$

The $x$ and $y$ components of the above equation take the following form

$$
\begin{gathered}
\frac{\partial^{2} U}{\partial y^{2}}-\frac{C_{0}^{2}}{K} U=\frac{1}{\mu_{\mathrm{f}}} \frac{\partial P}{\partial x} \\
V-\dot{h}=-\frac{K}{\mu_{\mathrm{f}}} \frac{\partial P}{\partial y}
\end{gathered}
$$

where $\dot{h}$ refers to the velocity with which the porous medium itself is moving and $C_{0}^{2}=1+M^{2} / \sigma^{2}$.

In (18) the effect of the viscous resistance term is neglected compared to the Darcy resistance because saturation sets in much faster in the transverse direction. Eliminating pressure between (17) and (18) we obtain:

$$
\frac{\partial^{4} U}{\partial y^{4}}-\frac{C_{0}^{2}}{K} \frac{\partial^{2} U}{\partial y^{2}}=\frac{1}{K} \frac{\partial^{2} U}{\partial x^{2}}
$$

This equation requires six boundary conditions; four on $y$ and two on $x$. The two boundary conditions on $y$ are given by equations (10) and (11).

Since the porous medium is bounded by a solid wall, $y=h+H$ at which $v=\dot{h}$, we have using (18),

$$
\frac{\partial P}{\partial y}=0 \text { at } y=h+H
$$

Equation (17), using (20), leads to 


$$
\frac{\partial^{3} U}{\partial y^{3}}-\frac{C_{0}^{2}}{K} \frac{\partial U}{\partial y}=0 \text { at } y=h+H
$$

The remaining boundary condition on $y$ can be obtained by matching the vertical component of velocity i.e.

$$
v_{h}=V(x, y) \text { at } y=h
$$

The two conditions on $x$ can be obtained from the pressure condition

$$
P=0 \text { at } x=0 \text { and } x=l
$$

The boundary condition (23) is used to derive the following boundary condition on $U$ with respect to $x$. With the help of equations (23), (18), (2) and the constitutive equations, we have

$$
\frac{\partial U}{\partial x}=0 \text { at } x=0 \text { and } x=l
$$

Assuming the solution for $U$ in the form

$$
U=F(y) G(x)
$$

and using the tránsformed boundary conditions

$$
\frac{\mathrm{d} G}{\mathrm{~d} x}=0 \text { at } x=0 \text { and } x=l
$$

and

$$
\begin{gathered}
F=0 \text { at } y=h+H \\
\frac{\mathrm{d} F}{\mathrm{~d} y}=-\frac{\alpha}{\sqrt{K}} F \text { at } y=h \\
\frac{\mathrm{d}^{3} F}{\mathrm{~d} y^{3}}-\frac{C_{0}^{2}}{K}\left(\frac{\mathrm{d} F}{\mathrm{~d} y}\right)=0 \text { at } y=h+H
\end{gathered}
$$

the complete solution for $U$ is obtained in the form: 


$$
\begin{aligned}
U= & \sum_{n=1}^{\infty} G_{n}\left[\mathrm{e}^{n_{1}(y / H)}+\left\{\left(\frac{E-D}{C}\right) \mathrm{e}^{\left(n_{1}+n_{3}\right)[(h / H)+1]}\right.\right. \\
& \left.+\frac{B}{A} \mathrm{e}^{\left(n_{1}+n_{4}\right)[(h / H)+1]}-\mathrm{e}^{\left.2 n_{1}(h / H)+1\right]}\right\} \mathrm{e}^{n_{2}(y / H)} \\
& \left.-\left(\frac{E-D}{C}\right) \mathrm{e}^{n_{3}(y / H)}-\frac{B}{A} \mathrm{e}^{n_{4}(y / H)}\right]
\end{aligned}
$$

where

$$
\begin{aligned}
& A=\frac{1}{H^{3}}\left[\bar{P} C \mathrm{e}^{n_{4}[(h / H)+1]}-R D^{\prime} \mathrm{e}^{n_{3}[(h / H)+1]}\right] \\
& B=\frac{1}{H^{3}}\left[R C \mathrm{e}^{n_{1}[(h / H)+1]}-E S_{0} \mathrm{e}^{n_{3}[(h / H)+1]}\right] \\
& \bar{P}=\left[\left(n_{2}^{3}-n_{4}^{3}\right)-C_{0}^{2} \frac{H^{2}}{K}\left(n_{2}-n_{4}\right)\right] \\
& S_{0}=\left[\left(n_{2}^{3}-n_{3}^{3}\right)-C_{0}^{2} \frac{H^{2}}{K}\left(n_{2}-n_{3}\right)\right] \\
& R=\left[\left(n_{2}^{3}-n_{1}^{3}\right)-C_{0}^{2} \frac{H^{2}}{K}\left(n_{2}-n_{1}\right)\right] \\
& \lambda_{n}=n \pi / \sqrt{K} l \\
& n_{1}=\sqrt{\frac{C_{0}^{2} \frac{H^{2}}{K}+\sqrt{C_{0}^{4} \frac{H^{4}}{K^{2}}-4 \lambda_{n}^{2}}}{2}} \\
& n_{2}=-n_{1} \\
& n_{3}=\sqrt{\frac{C_{0}^{2} \frac{H^{2}}{K}-\sqrt{C_{0}^{4} \frac{H^{4}}{K^{2}}}-4 \lambda_{n}^{2}}{2}} \\
& n_{4}=-n_{3} \\
& C=\frac{1}{H}\left[\left(n_{2}+\alpha-\frac{H}{\sqrt{K}}\right) \mathrm{e}^{n_{3}[(h / H)+1]}-\left(n_{3}+\alpha \frac{H}{\sqrt{K}}\right) \mathrm{e}^{n_{3}(h / H)+n_{2}}\right]
\end{aligned}
$$




$$
\begin{gathered}
D^{\prime}=\frac{1}{H}\left[\left(n_{2}+\alpha \frac{H}{\sqrt{K}}\right) \mathrm{e}^{n_{4}[(h / H)+1]}-\left(n_{4}+\alpha \frac{H}{\sqrt{K}}\right) \mathrm{e}^{n_{1}(h / H)+n_{2}}\right] \\
E=\frac{1}{H}\left[\left(n_{2}+\alpha \frac{H}{\sqrt{K}}\right) \mathrm{e}^{n_{1}[(h / H)+1]}-\left(n_{1}+\alpha \frac{H}{\sqrt{K}}\right) \mathrm{e}^{n_{1}\left(h_{1} / H\right)+n_{2}}\right] \\
D=\frac{B}{A} D^{\prime}
\end{gathered}
$$

The series coefficients $G_{n}$ appearing in (27) is still an unknown. Before determing $G_{n}$, we obtain an expression for $P$, the pressure in the porous medium making use of equations (27) and (17). The expression for pressure $P$ takes the form

$$
\begin{aligned}
P= & \sum_{n=1}^{\infty} \mu_{\mathrm{f}} \frac{G_{n}}{H^{2}}\left[\left(n_{1}^{2}-C_{0}^{2} \frac{H^{2}}{K}\right) \mathrm{e}^{n_{1}(y / H)}+\left(n_{2}^{2}-C_{0}^{2} \frac{H^{2}}{K}\right)\right. \\
& \cdot\left\{\left(\frac{E-D}{C}\right) \mathrm{e}^{\left(n_{1}+n_{3}\right)[(h / H)+1]}+\frac{B}{A} \mathrm{e}^{\left(n_{1}+n_{4}\right)[(h / H)+1]}\right. \\
& \left.-\mathrm{e}^{2 n_{1}[(h / H)+1]}\right\} \mathrm{e}^{n_{2}(y / H)}-\left(\frac{E-D}{C}\right)\left(n_{3}^{2}-C_{0}^{2} \frac{H^{2}}{K}\right) \mathrm{e}^{n_{3}(y / H)} \\
& \left.-\frac{B}{A}\left(n_{4}^{2}-C_{0}^{2} \frac{H^{2}}{K}\right) \mathrm{e}^{n_{4}(y / H)}\right]\left[\frac{l}{n \pi} \sin \frac{n \pi x}{l}\right]
\end{aligned}
$$

The expression for $\dot{h}$ is obtained using the continuity of the transverse velocity

$$
v_{h}=V(x, h)
$$

and the continuity of pressure $p(x)=P(x, h)$, in the form

$$
\frac{h^{\prime} l^{2}}{h^{3}}=-G_{n} Y \frac{l}{n \pi} \sin \left(\frac{n \pi x}{l}\right) \text {. }
$$

where

$$
\begin{aligned}
Y= & \frac{1}{A H^{2}}\left[\mathrm { e } ^ { - n _ { 1 } } \left\{\left(\left(M_{2} T_{1}-M_{1} T_{2}\right) \bar{P}+\left(M_{1} T_{2}-M_{2} T_{1}\right) S_{0}\right.\right.\right. \\
& \left.+\left(M_{3} T_{4}-M_{4} T_{3}\right) R\right) \mathrm{e}^{-n_{3}}+S_{0}\left(M_{4} T_{1}-M_{1} T_{4}\right) \mathrm{e}^{-n_{1}}
\end{aligned}
$$




$$
\begin{aligned}
& \left.+\bar{P}\left(M_{1} T_{3}-M_{3} T_{1}\right) \mathrm{e}^{-n_{1}-2 n_{3}}\right\}+\mathrm{e}^{-n_{3}}\left\{\left(\left(M_{3} T_{2}-M_{2} T_{3}\right) \bar{P}\right.\right. \\
& \left.\left.+\left(M_{2} T_{3}-M_{3} T_{2}\right) R\right) \mathrm{e}^{-n_{3}}\right\}+\left(M_{4} T_{2}-M_{2} T_{4}\right) R \\
& \left.+\left(M_{2} T_{4}-M_{4} T_{2}\right) S_{0}\right] \mathrm{e}^{n_{1}[(h / H)+1]+n_{3}} \\
& M_{i}=n_{i}+\alpha \sigma \frac{H}{h} \text { for } i=1,2,3,4 \\
& T_{i}=S \frac{l^{2}}{H^{2}} \frac{1}{H^{2} / K} n_{i}^{6}-2 C_{0}^{2} S \frac{l^{2}}{H^{2}} n_{i}^{4}+C_{0}^{4} S \frac{l^{2}}{H^{2}} \frac{H^{2}}{K} n_{i}^{2} \\
& -\frac{K l}{h^{3}} \frac{l}{H} n_{i}^{3}+C_{0}^{2} \frac{K l}{h^{3}} \frac{l}{H} \frac{H^{2}}{K} n_{i} \\
& \quad \text { for } i=1,2,3,4
\end{aligned}
$$

Now, the series constants $G_{n}$ are determined making use of the orthogonality of the functions $\sin (n \pi x / l)$.

Thus,

$$
G_{2 n+1}=\frac{4 \dot{h} l}{Y}
$$

where $n$ takes the values $0,1,2,3, \ldots$ Since $G_{n}$ is now known, the pressure $P$ in the porous medium given by $(28)$ is completely determined. So also the velocities $U$ and $V$. Finally, the pressure in the squeeze film using the continuity condition $p(x)=P(x, h)$ takes the form as:

$$
\frac{h^{3}}{\mu_{\mathrm{f}} \dot{h}^{3}} p(x)=\frac{4}{(2 n+1) \pi} \frac{X}{Y} \sin \left\{\frac{(2 n+1) \pi x}{l}\right\}
$$

where

$$
\begin{aligned}
X= & \frac{1}{A H^{2}}\left[\mathrm { e } ^ { - n _ { 1 } } \left\{\left(\left(M_{2} N_{1}^{2}-M_{1} N_{2}^{2}\right) \bar{P}+\left(M_{1} N_{2}^{2}-M_{2} N_{1}^{2}\right) S_{0}\right.\right.\right. \\
& \left.+\left(M_{3} N_{4}^{2}-M_{4} N_{3}^{2}\right) R\right) \mathrm{e}^{-n_{3}}+S_{0}\left(M_{4} N_{1}^{2}-M_{1} N_{4}^{2}\right) \mathrm{e}^{-n_{1}} \\
& \left.+\bar{P}\left(M_{1} N_{3}^{2}-M_{3} N_{1}^{2}\right) \mathrm{e}^{-n_{1}-2 n_{3}}\right\}+\mathrm{e}^{-n_{3}}\left\{\left(\left(M_{3} N_{2}^{2}-M_{2} N_{3}^{2}\right) \bar{P}\right.\right. \\
& \left.\left.+\left(M_{2} N_{3}^{2}-M_{3} N_{2}^{2}\right) R\right) \mathrm{e}^{-n_{3}}\right\}+\left(M_{4} N_{2}^{2}-M_{2} N_{4}^{2}\right) R \\
& \left.+\left(M_{2} N_{4}^{2}-M_{4} N_{2}^{2}\right) S_{0}\right] \mathrm{e}^{n_{1}[(h / H)+1]+n_{3}}
\end{aligned}
$$




$$
N_{i}^{2}=n_{i}^{2}-C_{0}^{2} \frac{H^{2}}{K} \text { for } i=1,2,3,4
$$

\section{\$ 5. Load capacity and thickness time relation}

The load per unit length in the $y$-direction which the film is capable of supporting is found by integrating (32) between $x=0$ to $x=l$ i.e.

$$
\text { Load capacity } L=\int_{0}^{l} p(x) \mathrm{d} x
$$

This in non-dimensional form using (32) becomes:

$$
-\frac{h^{3}}{\mu_{\mathrm{f}} \dot{h} l^{3}} L=\sum_{n=0}^{\infty} \frac{8}{(2 n+1)^{2} \pi^{2}} \frac{X}{Y}
$$

The thickness time relation can be obtained for a constant load by integrating (34) from $h_{0}$ to $h$. Thus, the non-dimensional thickness time $t$ can be written in the form

$$
\frac{L h_{0}^{2}}{\mu_{\mathrm{f}} l^{3}} t=-\sum_{n=0}^{\infty} F_{n} I_{n}\left(h / h_{0}\right)
$$

where

$$
\begin{gathered}
F_{n}=\frac{8}{(2 n+1)^{2} \pi^{2}} \\
I_{n}\left(h / h_{0}\right)=\int_{1}^{h / h_{0}}\left[\frac{1}{\xi^{3}} \frac{X_{1}}{Y_{1}}\right] \mathrm{d} \xi \\
C_{0}^{2}=1+\frac{1}{\xi^{2}} \frac{M^{2}}{\sigma^{2}} \\
X_{1}=\frac{1}{A H^{2}}\left[\mathrm { e } ^ { - n _ { 1 } } \left\{\left(\left(Z_{2} N_{1}^{2}-Z_{1} N_{2}^{2}\right) \bar{P}+\left(Z_{1} N_{2}^{2}-Z_{2} N_{1}^{2}\right) S_{0}\right.\right.\right. \\
\left.+\left(Z_{3} N_{4}^{2}-Z_{4} N_{3}^{2}\right) R\right) \mathrm{e}^{-n_{3}}+S_{0}\left(Z_{4} N_{1}^{2}-Z_{1} N_{4}^{2}\right) \mathrm{e}^{-n_{1}} \\
\left.+\bar{P}\left(Z_{1} N_{3}^{2}-Z_{3} N_{1}^{2}\right) \mathrm{e}^{-n_{1}-2 n_{3}}\right\}+\mathrm{e}^{-n_{3}}\left\{\left(\left(Z_{3} N_{2}^{2}-Z_{2} N_{3}^{2}\right) \bar{P}\right.\right. \\
\left.\left.+\left(Z_{2} N_{3}^{2}-Z_{3} N_{2}^{2}\right) R\right) \mathrm{e}^{-n_{3}}\right\}+\left(Z_{4} N_{2}^{2}-Z_{2} N_{4}^{2}\right) R \\
\left.+\left(Z_{2} N_{4}^{2}-Z_{4} N_{2}^{2}\right) S_{0}\right] \mathrm{e}^{n_{1}[(h / H)+1]+n_{3}}
\end{gathered}
$$




$$
\begin{aligned}
& Y_{1}=\frac{1}{A H^{2}}\left[\mathrm { e } ^ { - n _ { 1 } } \left\{\left(\left(Z_{2} W_{1}-Z_{1} W_{2}\right) \bar{P}+\left(Z_{1} W_{2}-Z_{2} W_{1}\right) S_{0}\right.\right.\right. \\
& \left.+\left(Z_{3} W_{4}-Z_{4} W_{3}\right) R\right) \mathrm{e}^{-n_{3}}+S_{0}\left(Z_{4} W_{1}-Z_{1} W_{4}\right) \mathrm{e}^{-n_{1}} \\
& \left.+\bar{P}\left(Z_{1} W_{3}-Z_{3} W_{1}\right) \mathrm{e}^{-n_{1}-2 n_{3}}\right\}+\mathrm{e}^{-n_{3}}\left\{\left(\left(Z_{3} W_{2}-Z_{2} W_{3}\right) \bar{P}\right.\right. \\
& \left.\left.+\left(Z_{2} W_{3}-Z_{3} W_{2}\right) R\right) \mathrm{e}^{-n_{3}}\right\}+\left(Z_{4} W_{2}-Z_{2} W_{4}\right) R \\
& \left.+\left(Z_{2} W_{4}-Z_{4} W_{2}\right) S_{0}\right] \mathrm{e}^{n_{1}[(h / H)+1]+n_{3}} \\
& Z_{i}=n_{i}+\alpha \frac{H}{\sqrt{K}} \text { for } i=1,2,3,4 \\
& W_{i}=S_{1} \frac{l^{2}}{H^{2}} \frac{1}{H^{2} / K} n_{i}^{6}-2 C_{0}^{2} S_{1} \frac{l^{2}}{H^{2}} n_{i}^{4}+C_{0}^{4} S_{1} \frac{l^{2}}{H^{2}} \frac{H^{2}}{K} n_{i}^{2} \\
& -\frac{1}{\xi^{3}} \frac{K l}{h_{0}^{3}} \frac{l}{H} n_{i}^{3}+\frac{C_{0}^{2}}{\xi} \frac{K l}{h_{0}^{3}} \frac{l}{H} \frac{H^{2}}{K} n_{i} \\
& \text { for } i=1,2,3,4 \\
& S_{1}=\frac{M \sinh M\left(1-\frac{\alpha h_{0} \xi}{\sqrt{K}}\right)-M^{2} \cosh M+2 \alpha \frac{h_{0} \xi}{\sqrt{K}}(\cosh M-1)}{M^{3}(M \cosh M+\alpha \sigma \sinh M)} .
\end{aligned}
$$

The above indicated integration in equation (37) was performed numerically in an I.B.M. 360 computer.

\section{§ 6. Results and conclusions}

Examination of the convergence of the series in equation (32) reveals that only three terms are needed to achieve accuracies sufficient for graphical presentation of the results but nevertheless ten terms are evaluated in all cases. The non-dimensional load capacity depends on the parameters $l / h$, $h / H, K l / h^{3}, 1 / \alpha \sigma, H^{2} / K$ and $M$. The dimensionless parameters $K l / h^{3}, 1 / \alpha \sigma$ and $H^{2} / K$ are referred to as the porous medium group, the slip group and the viscous group. The results of the present investigation are presented in Figs., 2 to 8 in which the curve $H^{2} / K \rightarrow \infty$ refers to the case of usual Darcy law. The viscous group is dominant when $H^{2} / K$ is of order 100 . In order to compare our results with the earlier work (Sparrow et al [7], Chandrasekhara [2]) we choose $l / h=1000$ and $h / H=0.1$ and the results are presented in Figs. 2 to 4 with the abscissa variable as the porous medium 


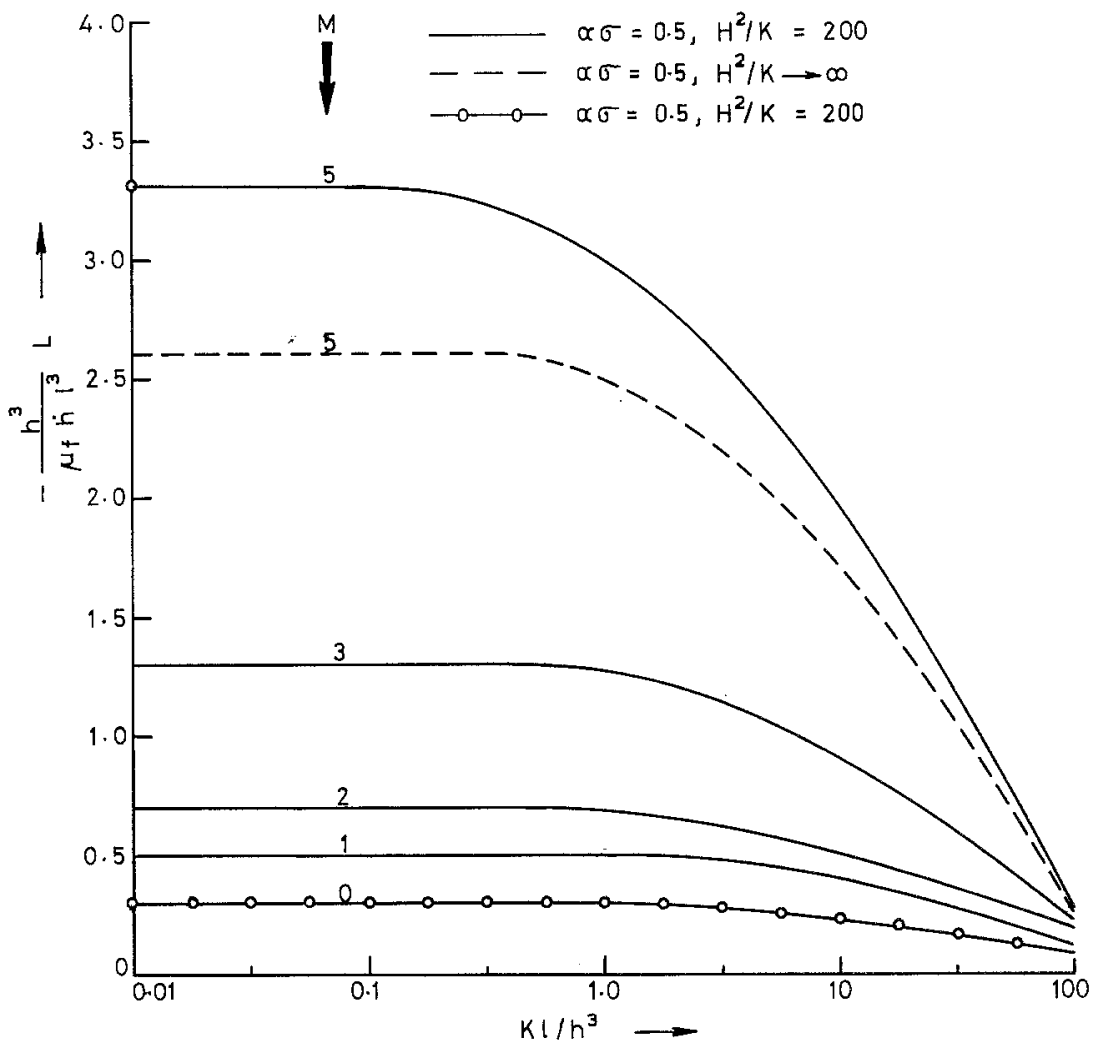

Fig. 2. Load-carrying capacity for different values of Hartmann number $M$.

group, with the Hartmann number $M$ and the viscous group $H^{2} / K$ as curve parameters. It is observed that increase in magnetic field increases the load capacity for a given value of the porous group. However, for a given value of $M$, increase in the value of the porous group decreases the load capacity. The higher the permeability, the more readily does the fluid flow through the porous material. In this way, the presence of the porous material decreases the resistance to the tangential flow and as a consequence the load carrying capacity is decreased.

It is further noticed that viscous resistance $H^{2} / K$ and magnetic field have the combined effect of increasing the load capacity. Also, for a given value of $M$ and small values of porous group $K l / h^{3}$, the presence of the viscous resistance increases the load capacity by a significant amount which is 


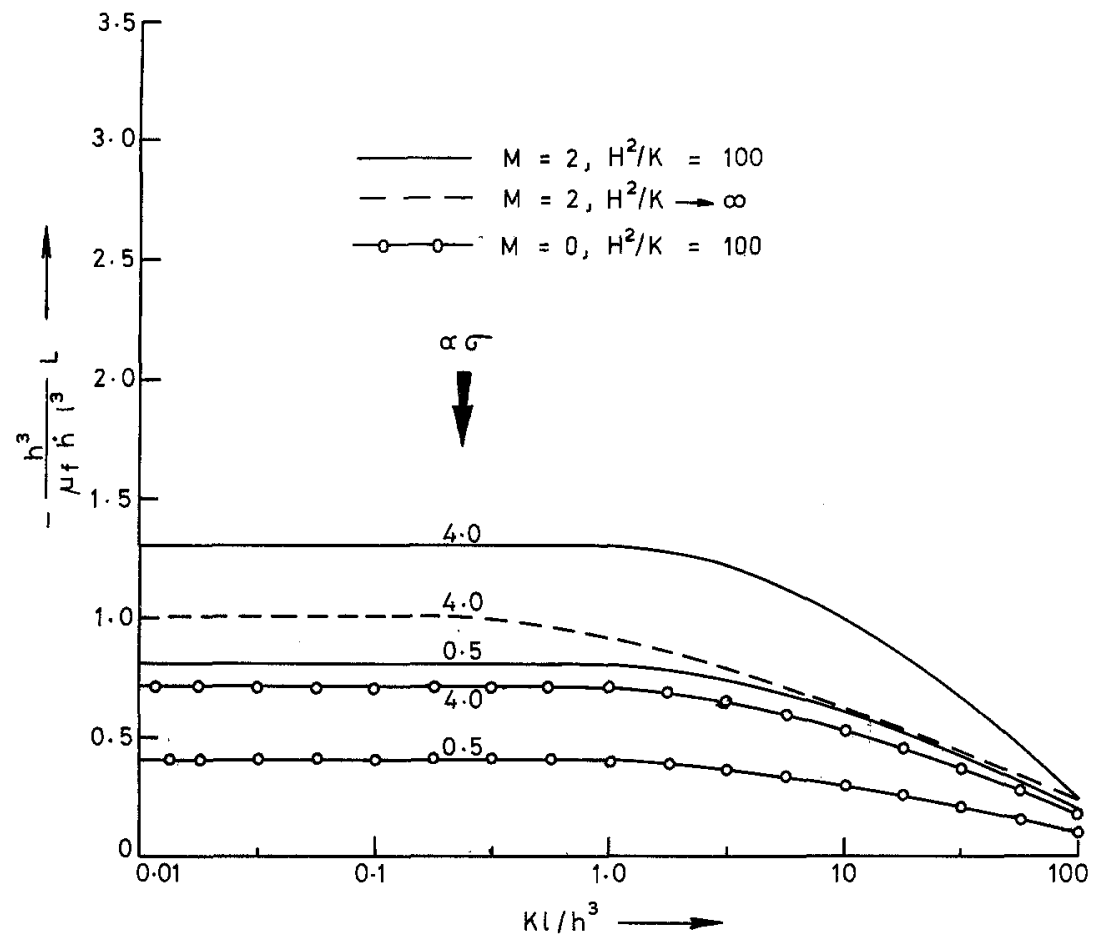

Fig. 3. Load-carrying capacity for different values of slip group $\alpha \sigma$.

evident from the dashed and solid curves $(M=5)$ in Fig. 2. But as $K l / h^{3}$ is increased, viscous resistance becomes ineffective and the effect of porous medium group becomes dominant and hence load capacity decreases. Fig. 3 depicts the variation of load capacity with the reciprocal of the slip group $\alpha \sigma$. It is clear from Fig. 3 that the effect of velocity slip is to decrease the resistance encountered by the fluid flowing in the gap itself and to diminish the load carrying capacity. Fig. 4 indicates that lower values of viscous group $H^{2} / K$ enhances the load capacity at moderate values of $\mathrm{Kl} / \mathrm{h}^{3}$.

Next we consider thickness-time results for squeeze films subject to constant loading and is presented in Figs. 5 to 8. In each figure, the dimensionless time group is plotted as a function of the ratio of instantaneous thickness $h$ to thickness $h_{0}$ at $t=0$. Fig. 5 shows that thickness 


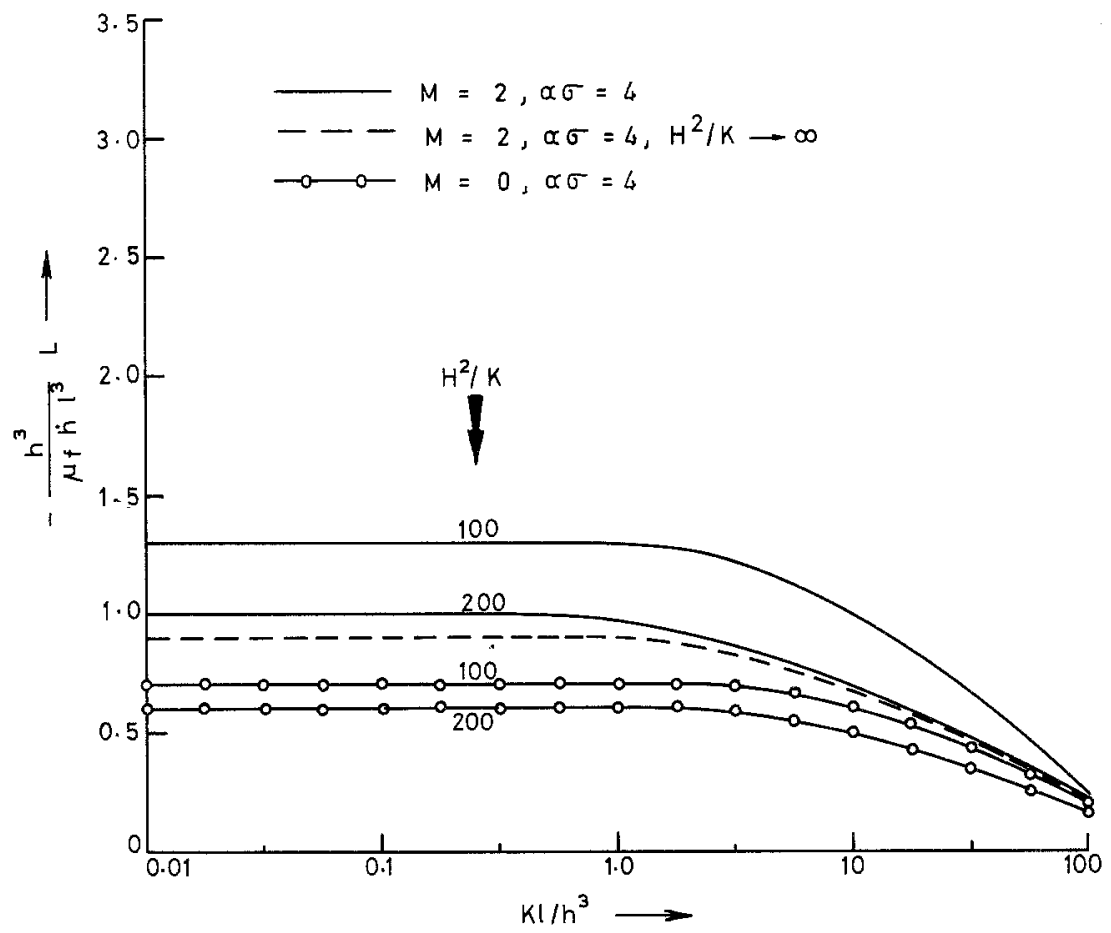

Fig. 4. Load carrying capacity for different values of $H^{2} / K$.

time in the presence of viscous resistance increases with $M$ and this is evident from the dashed and solid curves in Fig. 5. Figs. 6 to 8 depict the behaviour of thickness time with respect to the slip group, viscous group, $H^{2} / K$ and with $K l / h_{0}^{3}$. All the graphs exhibit the same common feature, that is the presence of viscous resistance and magnetic field increases the time required to attain a given value of $h / h_{0}$. Whereas increase in the porous medium group decreases the thickness time.

The results presented demonstrate that viscous resistance together with the magnetic field increases the load capacity. To obtain higher load capacity and substantial gain in response of squeeze films a suitable choice of porous material has to be made. 


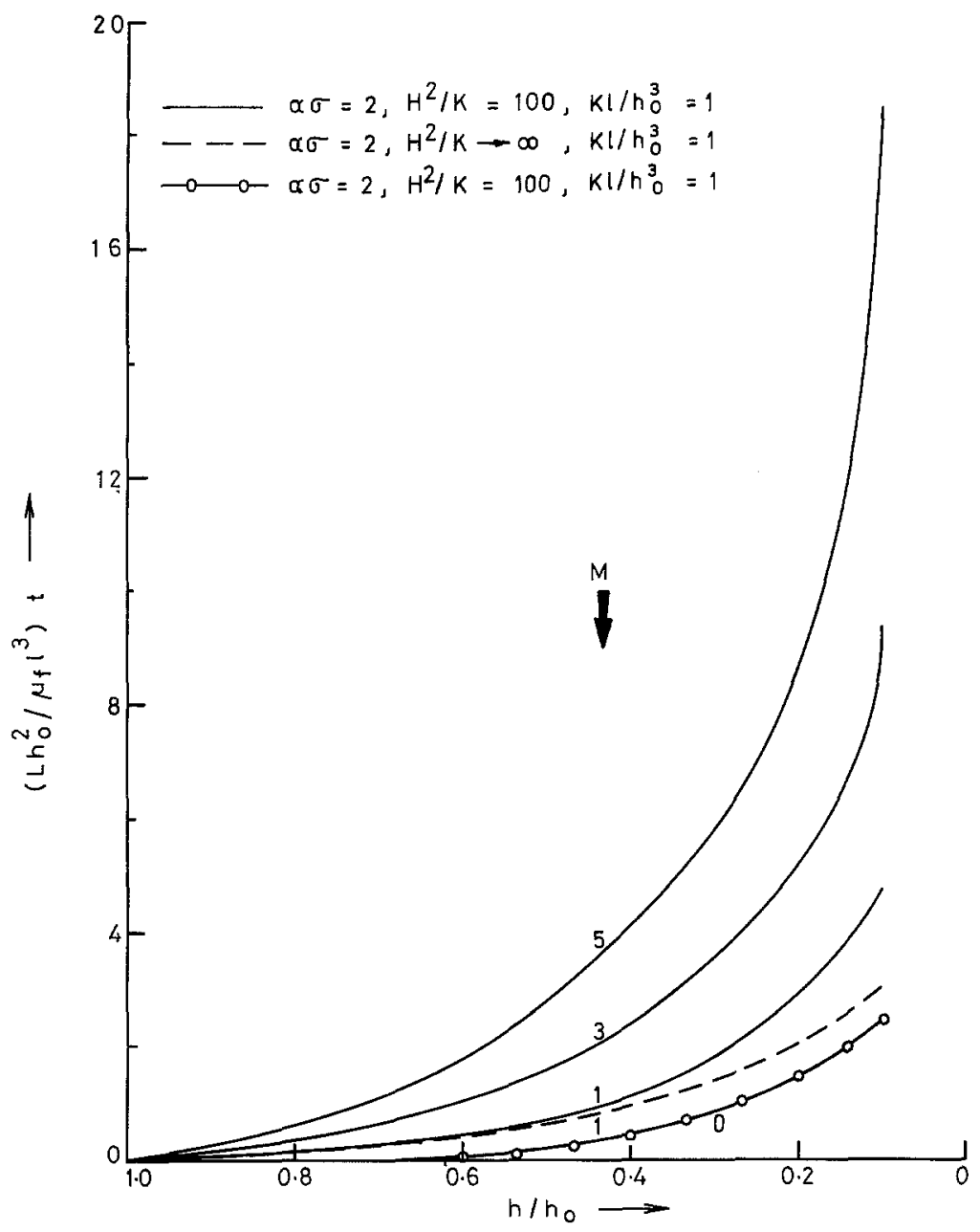

Fig. 5. Thickness-time relation for different values of $M$. 


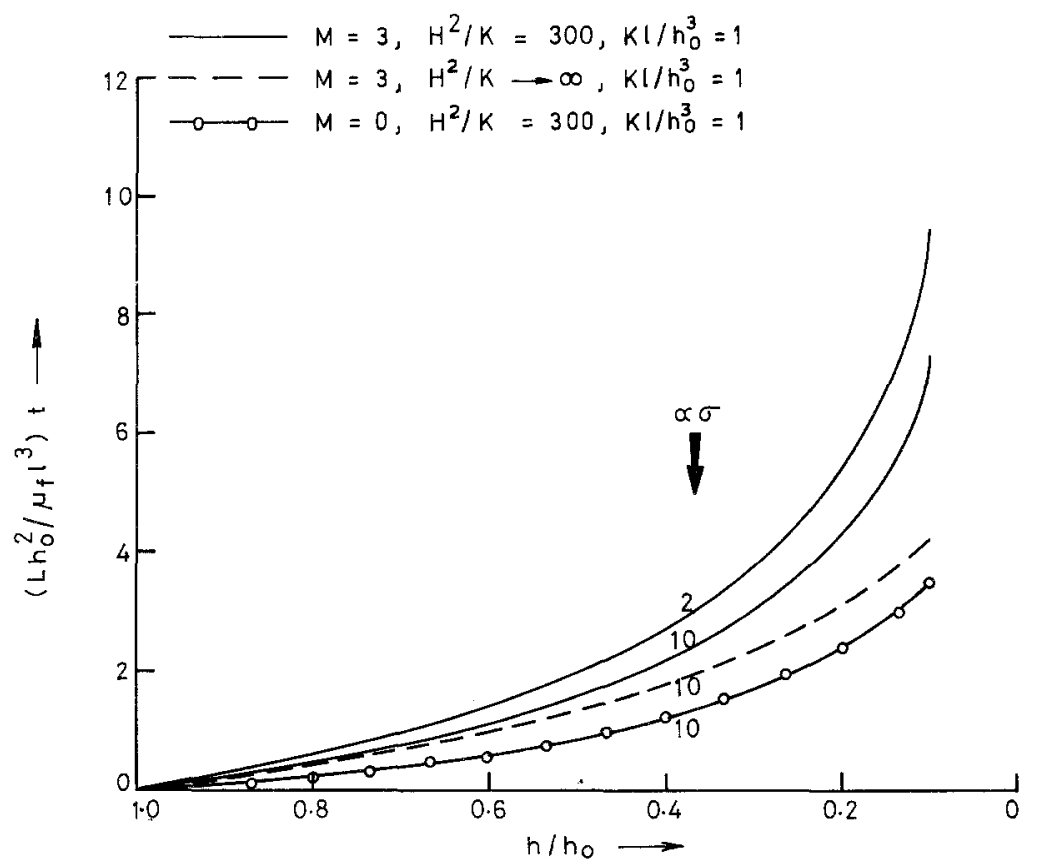

Fig. 6. Thickness-time relation for different values of $\alpha \sigma$.

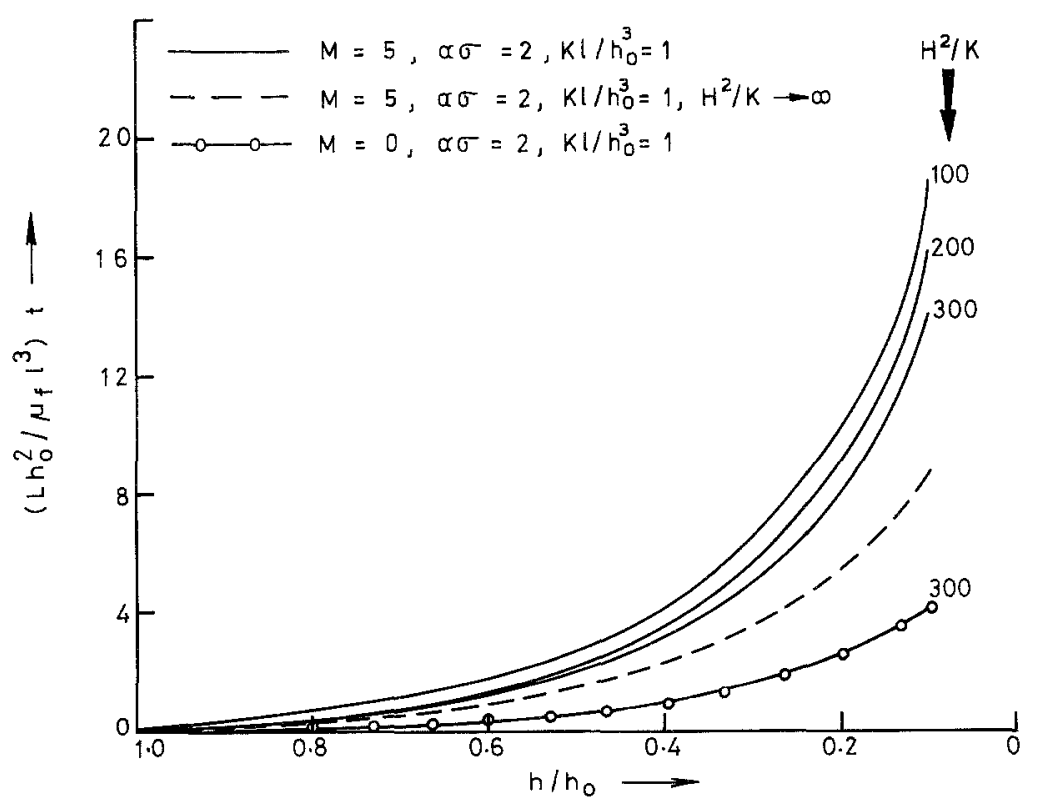

Fig. 7. Thickness-time relation for different values of $H^{2} / K$. 


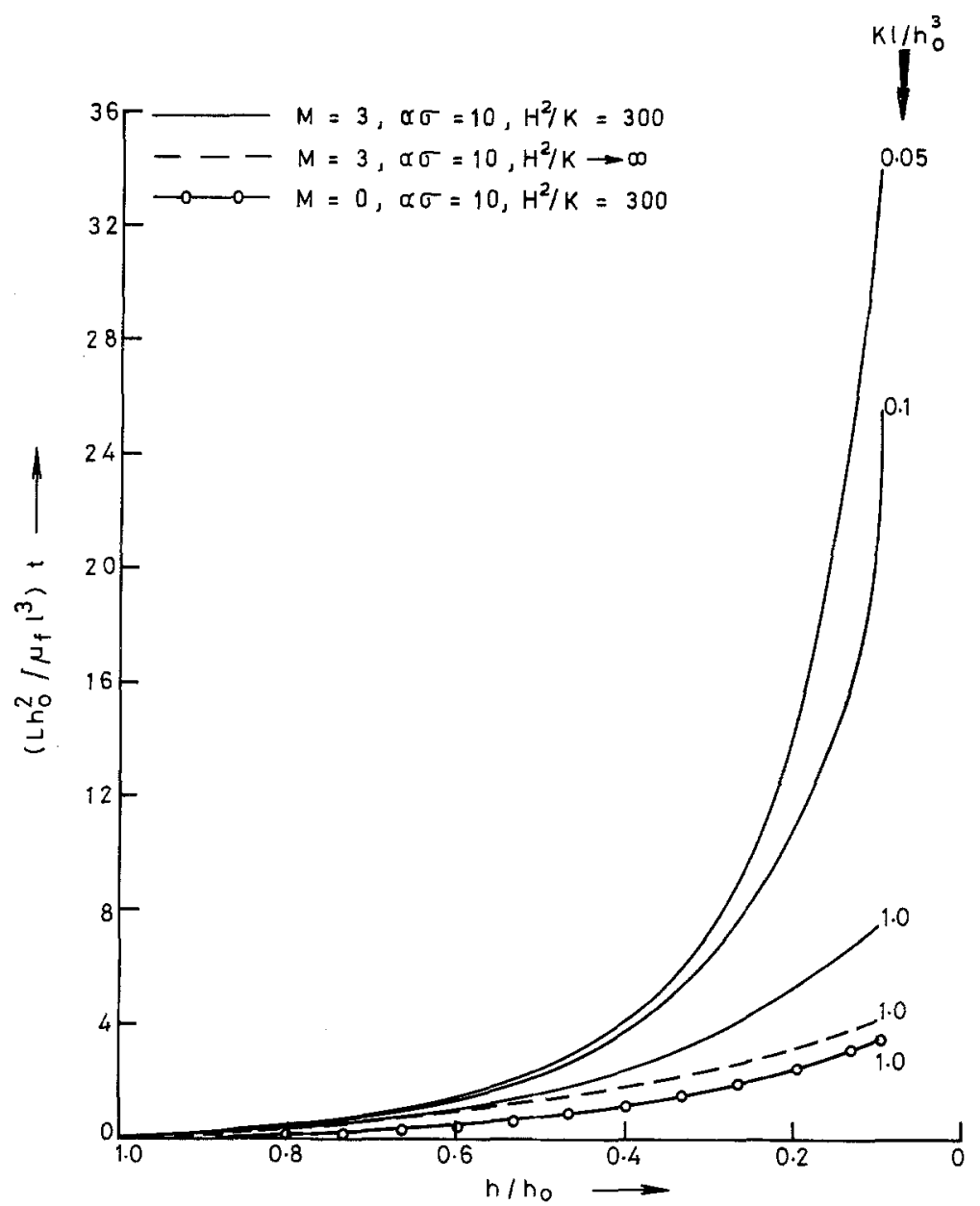

Fig. 8. Thickness-time relation for different values of $\mathrm{Kl} / h_{0}^{3}$.

\section{Acknowledgement}

The authors thank the referee for many valuable suggestions which improved the value of the paper.

The authors are very grateful to the Indian National Science Academy for sponsoring this work under the research project No. /31/BS/P-18/4521.

Received 2 March 1978

In final form 22 June 1978 


\section{REFERENCES}

[1] Beavers, G. S. and D. D. JosePh, J. Fluid Mech. 30 (1967) 197.

[2] Chandrasekhara, B. C., Appl. Sci. Res. 31 (1975) 52.

[3] MOORE, D. F., WEAR. 8 (1965) 245.

[4] HAYS, D. E., J. Basic Engg., Trans ASME, Series D, 85, No. 2 (1968) 243.

[5] JaCkson, J. D., Appl. Sci. Res. A11 (1963) 148.

[6] Gould, P., J. Lub. Tech., Trans. ASME 89 (1967) 375.

[7] Sparrow, E. M., G. S. Beavers, and I. T. Hwang, J. Lub. Tech., Trans. ASME 94 (1972) 260.

[8] Brinkman, H. C., Appl. Sci. Res. A1 (1947) 27.

[9] Rudraiah, N., Vignana Bharathi (Bangalore Univ. J.) 2 No. 2 (1976) 1.

[10] Rudratak, N. and S. T. Nagaraj, Int. J. of Engg. Sci. 15 (1977) 589.

[11] Taylor, G. I., J. Fluid Mech. 49 (1971) 319.

[12] Rudrajai, N., B. K. Ramaiah, and B. M. Rajasekhara, Int. J. Engg. Sci. 13(1975) 1.

[13] Rudraiah, N. and R. Patil, J. Appl. Mech. 40 (1973) 879. 